\title{
Analytical Solution for a Periodic Boundary Random-value Problem Via Stochastic Fixed Points with PPF Dependence Technique
}

\author{
Hasanen A. Hammad ${ }^{1, *}$, Rashwan A. Rashwan ${ }^{2}$ \\ ${ }^{1}$ Department of Mathematics, Faculty of Science, Sohag University, Sohag 82524, Egypt \\ 2 Department of Mathematics, Faculty of Science, Assuit University, Assuit 71516, Egypt
}

\begin{abstract}
In this paper, some random common fixed point and coincidence point theorems are established with PPF dependence for generalized random contractions in a separable Banach space. Our results introduce stochastic versions and extensions of recent results as $[3,21,25]$ and others. In addition, an application to establish PPF dependent solution of a periodic boundary random-valued problem is given to illustrate the usability of obtained results.
\end{abstract}

Keywords PPF dependent Random fixed point with a PPF dependent, Random generalized contractive mappings, The Razumikhin class.

AMS 2010 subject classifications 65Q20,47H04, 55M02.

DOI: $10.19139 /$ soic-2310-5070-511

\section{Introduction}

Fixed point theorem with the PPF (past, present and future) dependence was initiated in the work of Bernfeld et al. [3], where they gave great results concerning with fixed point for the mappings which are different domains and ranges. Also, they introduced the notion of Banach type contraction and proved some important results under this contraction. Many authors worked in this direction and obtained common fixed point for pair or families of contractive mappings in metric and abstract space (see $[2,5,6,8,9,10,14,18,19])$ and others. These results are useful for proving the existence solutions for certain functional differential equations.

Nice work presented by Spacek [26] and Hans [12] for studying random fixed point theorems in abstract spaces, they initiated this idea to get stochastic generalizations of the deterministic fixed point theorems in separable Banach spaces. After that, Bharucha-Reid [4] introduced his paper which gave power for this theory, he attracted the attention of several mathematicians and expanded this theory. So several random fixed point theorems have been obtained in the literature. Random fixed point theorems are useful for proving the existence results for random solutions of nonlinear random equations in separable Banach spaces (see [17, 22, 23, 24]) and others. A common assumption among all these operators in question to map an abstract space into itself, i.e. the domain and the range of the operators are the same.

Recently, Dhage [7] proved two basic random fixed point theorems for the operators in separable Banach spaces with PPF dependence and apply them to some nonlinear random differential equations for proving the existence and uniqueness of PPF dependent random solutions. After that, Hussian et al. [16] generalized and extended this results in the same space satisfying generalized random contractive conditions of Ćirić type.

\footnotetext{
*Correspondence to: Hasanen A. Hammad (Email: h_elmagd89@yahoo.com). Department of Mathematics, Faculty of Science, Sohag University, Sohag 82524, Egypt.
}

ISSN 2310-5070 (online) ISSN 2311-004X (print)

Copyright (C) 2019 International Academic Press 


\section{Preliminaries}

Let $E$ be a Banach space with the norm $\|\cdot\|_{E}$ and given a closed interval $I=[a, b]$ in $\mathbb{R}$, we consider the Banach space $E_{\circ}=C(I, E)$ of continuous $E$-valued functions defined on $I$, equipped with the norm $\|\cdot\|_{E_{\circ}}$ defined by

$$
\|y\|_{E_{\circ}}=\sup _{t \in I}\|y(t)\|,
$$

for all $y \in E_{\circ}$. For a fixed element $c \in I$, the Razumikhin or minimal class of functions in $E_{\circ}$ is defined by

$$
\Re_{c}=\left\{\phi \in E_{\circ}:\|\phi\|_{E_{\circ}}=\|\phi(c)\|_{E}\right\} .
$$

It's obvious that, every constant function from $I$ to $E$ belongs to $\Re_{c}$.

The class $\Re_{c}$ is algebraically closed with respect to the difference if $\xi-\eta \in \Re_{c}$ when $\xi, \eta \in \Re_{c}$, similarly, $\Re_{c}$ is topologically closed with respect to the topology on $E_{\circ}$ generated by the norm $\|\cdot\|_{E_{\circ}}$.

A mapping $\xi \in E_{\circ}$ is said to be a PPF dependent fixed point [3] or a fixed point with PPF dependence of mapping $T: E_{\circ} \rightarrow E$ if $T(\xi)=\xi(c)$ for some $c \in I$.

The following definition introduced in [25]:

Let $T, S: E_{\circ} \rightarrow E$ be two operators. A point $\zeta \in E_{\circ}$ is called

(i) PPF dependent common fixed point of $T$ and $S$ if $T(\zeta)=S(\zeta)=\zeta(c)$ for some $c \in I$.

(ii) PPF dependent coincidence point of $T$ and $S$ if $T(\zeta(c))=S(\zeta)$ for some $c \in I$.

An operator $T: E_{\circ} \rightarrow E$ is called Banach type contraction [3], if there is a real number $0 \leq \alpha<1$ such that

$$
\|T(\xi)-T(\eta)\|_{E} \leq \alpha\|\xi-\eta\|_{E_{\circ}}
$$

for all $\xi, \eta \in E_{\circ}$.

Geraghty [11] generalized Banach type contraction by introducing the following theorem:

Theorem 1

Let $(X, d)$ be a complete metric space, and $T: X \rightarrow X$ be an operator. Consider there exists $\beta:[0,+\infty) \rightarrow[0,1)$ satisfying the condition

$$
\beta\left(t_{n}\right) \rightarrow 1 \text { implies } t_{n} \rightarrow 0 \text { as } n \rightarrow+\infty .
$$

If $T$ satisfies the following inequality

$$
d(T x, T y) \leq \beta(d(x, y)) d(x, y), \text { for all } x, y \in X,
$$

then $T$ has a unique fixed point.

Recently, Parvaneh et al. [3] extended the results of Geraghty by giving the notion of a hybrid rational Geraghty type contraction as follows:

The mapping $T: E_{\circ} \rightarrow E$ is called hybrid rational Geraghty type contraction if

$$
\|T(\xi)-T(\eta)\|_{E} \leq \beta\left(\|\xi-\eta\|_{E_{\circ}}\right) M(\xi, \eta)+\gamma\left(\|\xi-\eta\|_{E_{\circ}}\right) N(\xi, \eta),
$$

for all $\xi, \eta \in E_{\circ}$, where $\gamma:[0, \infty) \rightarrow[0, \infty)$ is a bounded function,

$$
M(\xi, \eta)=\max \left\{\|\xi-\eta\|_{E_{\circ}}, \frac{\|\xi(c)-T(\xi)\|_{E}\|\eta(c)-T \eta\|_{E}}{1+\|T \xi-T \eta\|_{E}}\right\}
$$

and

for some $c \in I$.

$$
N(\xi, \eta)=\min \left\{\|\xi(c)-T \xi\|_{E},\|\eta(c)-T \eta\|_{E},\|\xi(c)-T \eta\|_{E},\|\eta(c)-T \xi\|_{E}\right\},
$$

Using the above definition Parvaneh et al. [21] proved some fixed point results with PPF dependence for Hybrid rational and Suzuki-Edelstein contractions.

Continuing in this line, we prove some common random fixed point results with PPF dependence for mappings in a separable Banach space satisfying general random contractions without the continuity. Our results generalize some known results in the literature. Finally an application is given to certain nonlinear functional random differential equations for proving the existence results for random solutions with a PPF dependent. 


\section{Random fixed point results with PPF dependence}

Let $(\Omega, X)$ be a measurable space and $E$ be a separable Banach space with norm $\|\cdot\|_{E}$. We equip the Banach space $E$ with a $\sigma$-algebra, $\chi_{E}$ of Borel subset of $E$ such that $\left(E, \chi_{E}\right)$ becomes a measurable space.

(i) A mapping $T: \Omega \rightarrow E$ is called measurable if

$$
T^{-1}(B)=\{\omega \in \Omega: T(\omega) \in B\} \in X
$$

for all Borel sets $B \in \chi_{E}$.

(ii) A mapping $T: \Omega \times E_{1} \rightarrow E_{2}$ is called random operator if $T(\omega, x)$ is measurable in $\omega$ for $x \in E_{1}$, where $E_{1}$ and $E_{2}$ are two Banach spaces and we denote a random operator $T$ on $E_{1}$ by $T(\omega) x=T(\omega, x)$.

(iii) A random operator $T(\omega)$ is called continuous in $E$ if $T(\omega, x)$ is continuous in $x$ for each $\omega \in \Omega$.

(iv) A measurable function $\xi^{*}: \Omega \rightarrow E_{\circ}$ is called a PPF dependent random fixed point of the random operator $T: \Omega \times E_{\circ} \rightarrow E$ if

$$
T\left(\omega, \xi^{*}(\omega)\right)=\xi^{*}(c, \omega)
$$

for some $c \in I$ and for all $\omega \in \Omega$.

Let $\digamma$ denote the class of all functions $\beta:[0,+\infty) \rightarrow[0,1)$ satisfy the following condition

$$
\beta\left(t_{n}\right) \rightarrow 1 \text { implies } t_{n} \rightarrow 0 \text { as } n \rightarrow+\infty .
$$

The random mapping $T: \Omega \times E_{\circ} \rightarrow E$ is called a hybrid rational Geraghty random contractive if there exists $\beta \in \digamma$ and $c \in I$ such that

$$
\|T(\omega, \xi)-T(\omega, \eta)\|_{E} \leq \beta\left(\|\xi(\omega)-\eta(\omega)\|_{E_{\circ}}\right) M(\xi(\omega), \eta(\omega))+\gamma\left(\|\xi(\omega)-\eta(\omega)\|_{E_{\circ}}\right) N(\xi(\omega), \eta(\omega)),
$$

for all $\xi, \eta \in E_{\circ}$, where $\gamma:[0, \infty) \rightarrow[0, \infty)$ is a bounded function,

$$
M(\xi(\omega), \eta(\omega))=\max \left\{\|\xi(\omega)-\eta(\omega)\|_{E_{\circ}}, \frac{\|\xi(c, \omega)-T(\omega, \xi(\omega))\|_{E}\|\eta(c, \omega)-T(\omega, \eta(\omega))\|_{E}}{1+\|T(\omega, \xi(\omega))-T(\omega, \eta(\omega))\|_{E}}\right\},
$$

and

$$
N(\xi(\omega), \eta(\omega))=\min \left\{\begin{array}{c}
\|\xi(c, \omega)-T(\omega, \xi(\omega))\|_{E},\|\eta(c, \omega)-T(\omega, \eta(\omega))\|_{E} \\
\|\xi(c, \omega)-T(\omega, \eta(\omega))\|_{E},\|\eta(c, \omega)-T(\omega, \xi(\omega))\|_{E}
\end{array}\right\}
$$

for all $\omega \in \Omega$.

Now, we present our first main results.

\section{Theorem 2}

Let $(\Omega, X)$ be a measurable space and let $E$ be a separable Banach space. If the operator $T: \Omega \times E_{\circ} \rightarrow E$ satisfy the condition of hybrid rational Geraghty random contractive (2) then the following statements hold in $E$ :

(a) If $\Re_{c}$ is algebraically closed with respect to difference, then for given $\xi_{\circ} \in E_{\circ}$ and $c \in I$, every sequence $\left\{\xi_{n}(\omega)\right\}$ of measurable functions satisfying

$$
T\left(\omega, \xi_{n-1}(\omega)\right)=\xi_{n}(c, \omega),
$$

and

$$
\left\|\xi_{n}(\omega)-\xi_{n+1}(\omega)\right\|_{E_{\circ}}=\left\|\xi_{n}(c, \omega)-\xi_{n+1}(c, \omega)\right\|_{E},
$$

for $n \in \mathbb{N}$ converges to a PPF dependent random fixed point of $T$.

(b) If $\Re_{c}$ is topologically closed, then for a given $\xi_{\circ} \in E_{\circ}$, every sequence $\left\{\xi_{n}(\omega)\right\}$ of iterates of $T$ constructed as in (a), converges to a unique PPF dependent random fixed point $\xi^{*}(\omega)$ of $T$.

Proof

Let $\xi_{\circ} \in E_{\circ}$ be arbitrary, by hypothesis $T\left(\omega, \xi_{\circ}(\omega)\right) \in E$, there exists $x_{1}(\omega)=T\left(\omega, \xi_{\circ}(\omega)\right)$, where the function 
$x_{1}: \Omega \rightarrow E$ is measurable. Choose a measurable function $\xi_{1}: \Omega \rightarrow E_{\circ}$ such that

$$
\left\|\xi_{1}(c, \omega)-\xi_{\circ}(c, \omega)\right\|_{E}=\left\|\xi_{1}(\omega)-\xi_{\circ}(\omega)\right\|_{E_{\circ}} .
$$

Continuing in this way, by induction, we have (3) and (4), i.e., $T\left(\omega, \xi_{n-1}(\omega)\right)=\xi_{n}(c, \omega)$ and

$$
\left\|\xi_{n-1}(c, \omega)-\xi_{n}(c, \omega)\right\|_{E}=\left\|\xi_{n-1}(\omega)-\xi_{n}(\omega)\right\|_{E_{\circ}},
$$

for all $n \in \mathbb{N}$ and $\omega \in \Omega$. By using (2), we get

$$
\begin{aligned}
\left\|\xi_{2}(\omega)-\xi_{3}(\omega)\right\|_{E_{\circ}}= & \left\|\xi_{2}(c, \omega)-\xi_{3}(c, \omega)\right\|=\left\|T\left(\omega, \xi_{1}(\omega)\right)-T\left(\omega, \xi_{2}(\omega)\right)\right\|_{E} \\
\leq & \beta\left(\left\|\xi_{1}(\omega)-\xi_{2}(\omega)\right\|_{E_{\circ}}\right) M\left(\xi_{1}(\omega), \xi_{2}(\omega)\right) \\
& +\gamma\left(\left\|\xi_{1}(\omega)-\xi_{2}(\omega)\right\|_{E_{\circ}}\right) N\left(\xi_{1}(\omega), \xi_{2}(\omega)\right)
\end{aligned}
$$

where,

$$
\begin{aligned}
M\left(\xi_{1}(\omega), \xi_{2}(\omega)\right) & =\max \left\{\left\|\xi_{1}(\omega)-\xi_{2}(\omega)\right\|_{E_{\circ}}, \frac{\left\|\xi_{1}(c, \omega)-T\left(\omega, \xi_{1}(\omega)\right)\right\|_{E}\left\|\xi_{2}(c, \omega)-T\left(\omega, \xi_{2}(\omega)\right)\right\|_{E}}{1+\left\|T\left(\omega, \xi_{1}(\omega)\right)-T\left(\omega, \xi_{2}(\omega)\right)\right\|_{E}}\right\} \\
& =\max \left\{\left\|\xi_{1}(\omega)-\xi_{2}(\omega)\right\|_{E_{\circ}}, \frac{\left\|\xi_{1}(c, \omega)-\xi_{2}(c, \omega)\right\|_{E}\left\|\xi_{2}(c, \omega)-\xi_{3}(c, \omega)\right\|_{E}}{1+\left\|\xi_{2}(c, \omega)-\xi_{3}(c, \omega)\right\|_{E}}\right\} \\
& \leq \max \left\{\left\|\xi_{1}(\omega)-\xi_{2}(\omega)\right\|_{E_{\circ}},\left\|\xi_{1}(c, \omega)-\xi_{2}(c, \omega)\right\|_{E}\right\} \\
& =\max \left\{\left\|\xi_{1}(\omega)-\xi_{2}(\omega)\right\|_{E_{\circ}},\left\|\xi_{1}(\omega)-\xi_{2}(\omega)\right\|_{E_{\circ}}\right\}=\left\|\xi_{1}(\omega)-\xi_{2}(\omega)\right\|_{E_{\circ}},
\end{aligned}
$$

and

$$
\begin{aligned}
N\left(\xi_{1}(\omega), \xi_{2}(\omega)\right) & =\min \left\{\begin{array}{c}
\left\|\xi_{1}(c, \omega)-T\left(\omega, \xi_{1}(\omega)\right)\right\|_{E},\left\|\xi_{2}(c, \omega)-T\left(\omega, \xi_{2}(\omega)\right)\right\|_{E}, \\
\left\|\xi_{1}(c, \omega)-T\left(\omega, \xi_{2}(\omega)\right)\right\|_{E},\left\|\xi_{2}(c, \omega)-T\left(\omega, \xi_{1}(\omega)\right)\right\|_{E}
\end{array}\right\} \\
& =\min \left\{\begin{array}{c}
\left\|\xi_{1}(c, \omega)-\xi_{2}(c, \omega)\right\|_{E},\left\|\xi_{2}(c, \omega)-\xi_{3}(c, \omega)\right\|_{E}, \\
\left\|\xi_{1}(c, \omega)-\xi_{3}(c, \omega)\right\|_{E},\left\|\xi_{2}(c, \omega)-\xi_{2}(c, \omega)\right\|_{E}
\end{array}\right\}=0 .
\end{aligned}
$$

From (5), we obtain

$$
\left\|\xi_{2}(\omega)-\xi_{3}(\omega)\right\|_{E_{\circ}} \leq \beta\left(\left\|\xi_{1}(\omega)-\xi_{2}(\omega)\right\|_{E_{\circ}}\right)\left\|\xi_{1}(\omega)-\xi_{2}(\omega)\right\|_{E_{\circ}}<\left\|\xi_{1}(\omega)-\xi_{2}(\omega)\right\|_{E_{\circ}} .
$$

Similarly, we can write

$$
\left\|\xi_{n}(\omega)-\xi_{n+1}(\omega)\right\|_{E_{\circ}} \leq \beta\left(\left\|\xi_{n-1}(\omega)-\xi_{n}(\omega)\right\|_{E_{\circ}}\right)\left\|\xi_{n-1}(\omega)-\xi_{n}(\omega)\right\|_{E_{\circ}}<\left\|\xi_{n-1}(\omega)-\xi_{n}(\omega)\right\|_{E_{\circ}},
$$

for all $n \in \mathbb{N}$. This meaning by that the sequence $\left\{\left\|\xi_{n}(\omega)-\xi_{n+1}(\omega)\right\|_{E_{0}}\right\}$ is decreasing in $\mathbb{R}_{+}$. So there exists $q \geq 0$ such that $\lim _{n \rightarrow+\infty}\left\|\xi_{n}(\omega)-\xi_{n+1}(\omega)\right\|_{E_{\circ}}=q$. If we consider $q>0$, then by taking the limit as $n \rightarrow+\infty$ in (6), we get

$$
q \leq \lim _{n \rightarrow+\infty} \beta\left(\left\|\xi_{n-1}(\omega)-\xi_{n}(\omega)\right\|_{E_{0}}\right) q
$$

This leads to $1 \leq \lim _{n \rightarrow+\infty} \beta\left(\left\|\xi_{n-1}(\omega)-\xi_{n}(\omega)\right\|_{E_{\circ}}\right)$. So

$$
\lim _{n \rightarrow+\infty} \beta\left(\left\|\xi_{n-1}(\omega)-\xi_{n}(\omega)\right\|_{E_{0}}\right)=1,
$$

since $\beta \in \digamma$ as in (1), then $\lim _{n \rightarrow+\infty}\left\|\xi_{n-1}(\omega)-\xi_{n}(\omega)\right\|_{E_{\circ}}=0$, which is a contradiction, so $q=0$ that is

$$
\lim _{n \rightarrow+\infty}\left\|\xi_{n}(\omega)-\xi_{n+1}(\omega)\right\|_{E_{\circ}}=0 .
$$

We claim that the measurable sequence $\left\{\xi_{n}(\omega)\right\}$ is a Cauchy sequence in $E_{\circ}$. Suppose the contrary, then we obtain

$$
\lim _{m, n \rightarrow+\infty}\left\|\xi_{n}(\omega)-\xi_{m}(\omega)\right\|_{E_{\circ}}>0
$$


By triangle inequality and since $T$ is a hybrid rational Geraghty random contractive mapping, one can write

$$
\begin{aligned}
& \left\|\xi_{n}(\omega)-\xi_{m}(\omega)\right\|_{E_{\circ}} \\
\leq & \left\|\xi_{n}(\omega)-\xi_{n+1}(\omega)\right\|_{E_{\circ}}+\left\|\xi_{n+1}(\omega)-\xi_{m+1}(\omega)\right\|_{E_{\circ}}+\left\|\xi_{m+1}(\omega)-\xi_{m}(\omega)\right\|_{E_{\circ}} \\
= & \left\|\xi_{n}(\omega)-\xi_{n+1}(\omega)\right\|_{E_{\circ}}+\left\|\xi_{m+1}(\omega)-\xi_{m}(\omega)\right\|_{E_{\circ}}+\left\|T\left(\omega, \xi_{n}(\omega)\right)-T\left(\omega, \xi_{m}(\omega)\right)\right\|_{E_{\circ}} \\
\leq & \left\|\xi_{n}(\omega)-\xi_{n+1}(\omega)\right\|_{E_{\circ}}+\left\|\xi_{m+1}(\omega)-\xi_{m}(\omega)\right\|_{E_{\circ}}+\beta\left(\left\|\xi_{n}(\omega)-\xi_{m}(\omega)\right\|_{E_{\circ}}\right) M\left(\xi_{n}(\omega), \xi_{m}(\omega)\right) \\
& +\gamma\left(\left\|\xi_{n}(\omega)-\xi_{m}(\omega)\right\|_{E_{\circ}}\right) M\left(\xi_{n}(\omega), \xi_{m}(\omega)\right) .
\end{aligned}
$$

Taking the limit as $m, n \rightarrow+\infty$ in the above inequality and applying (7), we observe that

$$
\begin{aligned}
& \lim _{m, n \rightarrow+\infty}\left\|\xi_{n}(\omega)-\xi_{m}(\omega)\right\|_{E_{\circ}} \\
\leq & \lim _{m, n \rightarrow+\infty} \beta\left(\left\|\xi_{n}(\omega)-\xi_{m}(\omega)\right\|_{E_{\circ}}\right) \lim _{m, n \rightarrow+\infty} M\left(\xi_{n}(\omega), \xi_{m}(\omega)\right) \\
+ & \lim _{m, n \rightarrow+\infty} \gamma\left(\left\|\xi_{n}(\omega)-\xi_{m}(\omega)\right\|_{E_{\circ}}\right) \lim _{m, n \rightarrow+\infty} N\left(\xi_{n}(\omega), \xi_{m}(\omega)\right),
\end{aligned}
$$

where

$$
\begin{aligned}
& \lim _{m, n \rightarrow+\infty} M\left(\xi_{n}(\omega), \xi_{m}(\omega)\right) \\
= & \lim _{m, n \rightarrow+\infty} \max \left\{\left\|\xi_{n}(\omega)-\xi_{m}(\omega)\right\|_{E_{\circ}}, \frac{\left\|\xi_{n}(c, \omega)-T\left(\omega, \xi_{n}(\omega)\right)\right\|_{E}\left\|\xi_{m}(\omega, c)-T\left(\omega, \xi_{m}(\omega)\right)\right\|_{E}}{1+\left\|T\left(\omega, \xi_{n}(\omega)\right)-T\left(\omega, \xi_{m}(\omega)\right)\right\|_{E}}\right\} \\
= & \lim _{m, n \rightarrow+\infty} \max \left\{\left\|\xi_{n}(\omega)-\xi_{m}(\omega)\right\|_{E_{\circ}}, \frac{\left\|\xi_{n}(c, \omega)-\xi_{n+1}(c, \omega)\right\|_{E}\left\|\xi_{m}(\omega, c)-\xi_{m+1}(\omega, c)\right\|_{E}}{1+\left\|\xi_{n+1}(\omega, c)-\xi_{m+1}(\omega, c)\right\|_{E}}\right\} \\
= & \lim _{m, n \rightarrow+\infty}\left\|\xi_{n}(\omega)-\xi_{m}(\omega)\right\|_{E_{\circ}},
\end{aligned}
$$

also

$$
\begin{aligned}
& \lim _{m, n \rightarrow+\infty} N\left(\xi_{n}(\omega), \xi_{m}(\omega)\right) \\
= & \lim _{m, n \rightarrow+\infty} \min \left\{\begin{array}{l}
\left\|\xi_{n}(c, \omega)-T\left(\omega, \xi_{n}(\omega)\right)\right\|_{E},\left\|\xi_{m}(c, \omega)-T\left(\omega, \xi_{m}(\omega)\right)\right\|_{E}, \\
\left\|\xi_{n}(c, \omega)-T\left(\omega, \xi_{m}(\omega)\right)\right\|_{E},\left\|\xi_{m}(c, \omega)-T\left(\omega, \xi_{n}(\omega)\right)\right\|_{E}
\end{array}\right\} \\
= & \lim _{m, n \rightarrow+\infty} \min \left\{\begin{array}{l}
\left\|\xi_{n}(c, \omega)-\xi_{n+1}(c, \omega)\right\|_{E},\left\|\xi_{m}(c, \omega)-\xi_{m+1}(c, \omega)\right\|_{E}, \\
\left\|\xi_{n}(c, \omega)-\xi_{m+1}(c, \omega)\right\|_{E},\left\|\xi_{m}(c, \omega)-\xi_{n+1}(c, \omega)\right\|_{E}
\end{array}\right\}=0 .
\end{aligned}
$$

Applying (9) and (10) in (8), it follows that

$$
\limsup _{m, n \rightarrow+\infty}\left\|\xi_{n}(\omega)-\xi_{m}(\omega)\right\|_{E_{\circ}} \leq \limsup _{m, n \rightarrow+\infty} \beta\left(\left\|\xi_{n}(\omega)-\xi_{m}(\omega)\right\|_{E_{\circ}}\right) \cdot \limsup _{m, n \rightarrow+\infty}\left\|\xi_{n}(\omega)-\xi_{m}(\omega)\right\|_{E_{\circ}}
$$

which mean $1 \leq \limsup _{m, n \rightarrow+\infty} \beta\left(\left\|\xi_{n}(\omega)-\xi_{m}(\omega)\right\|_{E_{0}}\right)$, that is

$$
\lim _{n, m \rightarrow+\infty} \beta\left(\left\|\xi_{n}(\omega)-\xi_{m}(\omega)\right\|_{E_{\circ}}\right)=1
$$

since $\beta \in \digamma$, we deduce

$$
\lim _{n, m \rightarrow+\infty}\left\|\xi_{n}(\omega)-\xi_{m}(\omega)\right\|_{E_{\circ}}=0
$$

which is a contradiction again. Therefore

$$
\lim _{n, m \rightarrow+\infty}\left\|\xi_{n}(\omega)-\xi_{m}(\omega)\right\|_{E_{\circ}}=0 .
$$


This shows that $\left\{\xi_{n}(\omega)\right\}$ is a Cauchy sequence of measurable function on $\Omega$ to $E_{\circ}$. Since $E_{\circ}$ is a separable Banach space hence it complete, there is a measurable function $\xi^{*}: \Omega \rightarrow E_{\circ}$ such that $\lim _{n \rightarrow+\infty} \xi_{n}(\omega)=\xi^{*}(\omega)$ for all $\omega \in \Omega$.

Now, we prove that $\xi^{*}(\omega)$ is a random fixed point with PPF dependence of the random operator $T$ on $E_{\circ}$. From (2), we get

$$
\begin{aligned}
\left\|T\left(\omega, \xi^{*}(\omega)\right)-\xi^{*}(c, \omega)\right\|_{E} \leq & \left\|T\left(\omega, \xi^{*}(\omega)\right)-\xi_{n}(c, \omega)\right\|_{E}+\left\|\xi_{n}(c, \omega)-\xi^{*}(c, \omega)\right\|_{E} \\
= & \left\|T\left(\omega, \xi^{*}(\omega)\right)-T\left(\omega, \xi_{n-1}(\omega)\right)\right\|_{E}+\left\|\xi_{n}(\omega)-\xi^{*}(\omega)\right\|_{E_{\circ}} \\
\leq & \beta\left(\left\|\xi^{*}(\omega)-\xi_{n-1}(\omega)\right\|_{E_{\circ}}\right) M\left(\xi^{*}(\omega), \xi_{n-1}(\omega)\right) \\
& +\gamma\left(\left\|\xi^{*}(\omega)-\xi_{n-1}(\omega)\right\|_{E_{\circ}}\right) N\left(\xi^{*}(\omega), \xi_{n-1}(\omega)\right)+\left\|\xi_{n}(\omega)-\xi^{*}(\omega)\right\|_{E_{\circ}} .
\end{aligned}
$$

Taking the limit as $n \rightarrow+\infty$ in the above inequality, yields

$$
\begin{aligned}
& \leq\left\|T\left(\omega, \xi^{*}(\omega)\right)-\xi^{*}(c, \omega)\right\|_{E} \\
& \lim _{n \rightarrow+\infty} \beta\left(\left\|\xi^{*}(\omega)-\xi_{n-1}(\omega)\right\|_{E_{\circ}}\right) \cdot \lim _{n \rightarrow+\infty} M\left(\xi^{*}(\omega), \xi_{n-1}(\omega)\right) \\
&+\lim _{n \rightarrow+\infty} \gamma\left(\left\|\xi^{*}(\omega)-\xi_{n-1}(\omega)\right\|_{E_{\circ}}\right) \cdot \lim _{n \rightarrow+\infty} N\left(\xi^{*}(\omega), \xi_{n-1}(\omega)\right) .
\end{aligned}
$$

But

$$
\begin{aligned}
& \lim _{n \rightarrow+\infty} M\left(\xi^{*}(\omega), \xi_{n-1}(\omega)\right) \\
= & \lim _{n \rightarrow+\infty} \max \left\{\left\|\xi^{*}(\omega)-\xi_{n-1}(\omega)\right\|_{E_{\circ}}, \frac{\left\|\xi^{*}(c, \omega)-T\left(\omega, \xi^{*}(\omega)\right)\right\|_{E}\left\|\xi_{n-1}(c, \omega)-T\left(\omega, \xi_{n-1}(\omega)\right)\right\|_{E}}{1+\left\|T\left(\omega, \xi^{*}(\omega)\right)-T\left(\omega, \xi_{n-1}(\omega)\right)\right\|_{E}}\right\} \\
= & \lim _{n \rightarrow+\infty} \max \left\{\left\|\xi^{*}(\omega)-\xi_{n-1}(\omega)\right\|_{E_{\circ}}, \frac{\left\|\xi^{*}(c, \omega)-T\left(\omega, \xi^{*}(\omega)\right)\right\|_{E}\left\|\xi_{n-1}(c, \omega)-\xi_{n}(c, \omega)\right\|_{E}}{1+\left\|T\left(\omega, \xi^{*}(\omega)\right)-\xi_{n}(c, \omega)\right\|_{E}}\right\} \\
= & \lim _{n \rightarrow+\infty} \max \left\{\left\|\xi^{*}(\omega)-\xi_{n-1}(\omega)\right\|_{E_{\circ}}, \frac{\left\|\xi^{*}(c, \omega)-T\left(\omega, \xi^{*}(\omega)\right)\right\|_{E}\left\|\xi_{n-1}(\omega)-\xi_{n}(\omega)\right\|_{E_{\circ}}}{1+\left\|T\left(\omega, \xi^{*}(\omega)\right)-\xi_{n}(c, \omega)\right\|_{E}}\right\}=0,
\end{aligned}
$$

and

$$
\begin{aligned}
& \lim _{n \rightarrow+\infty} N\left(\xi^{*}(\omega), \xi_{n-1}(\omega)\right) \\
= & \lim _{m, n \rightarrow+\infty} \min \left\{\begin{array}{c}
\left\|\xi^{*}(c, \omega)-T\left(\omega, \xi^{*}(\omega)\right)\right\|_{E},\left\|\xi_{n-1}(c, \omega)-T\left(\omega, \xi_{n-1}(\omega)\right)\right\|_{E}, \\
\left\|\xi^{*}(c, \omega)-T\left(\omega, \xi_{n-1}(\omega)\right)\right\|_{E},\left\|\xi_{n-1}(c, \omega)-T\left(\omega, \xi^{*}(\omega)\right)\right\|_{E}
\end{array}\right\} \\
= & \lim _{n \rightarrow+\infty} \min \left\{\begin{array}{c}
\left\|\xi^{*}(c, \omega)-T\left(\omega, \xi^{*}(\omega)\right)\right\|_{E},\left\|\xi_{n-1}(c, \omega)-\xi_{n}(c, \omega)\right\|_{E}, \\
\left\|\xi^{*}(c, \omega)-\xi_{n}(c, \omega)\right\|_{E},\left\|\xi_{n-1}(c, \omega)-T\left(\omega, \xi^{*}(\omega)\right)\right\|_{E}
\end{array}\right\} \\
= & \lim _{n \rightarrow+\infty} \min \left\{\begin{array}{c}
\left\|\xi^{*}(c, \omega)-T\left(\omega, \xi^{*}(\omega)\right)\right\|_{E},\left\|\xi_{n-1}(\omega)-\xi_{n}(\omega)\right\|_{E}, \\
\left\|\xi^{*}(\omega)-\xi_{n}(\omega)\right\|_{E_{\circ}},\left\|\xi_{n-1}(c, \omega)-T\left(\omega, \xi^{*}(\omega)\right)\right\|_{E}
\end{array}\right\}=0 .
\end{aligned}
$$

Applying (12) and (13) in (11), we deduce

$$
\left\|T\left(\omega, \xi^{*}(\omega)\right)-\xi^{*}(c, \omega)\right\|_{E}=\left\|T\left(\omega, \xi^{*}(\omega)\right)-\xi^{*}(\omega)\right\|_{E_{\circ}}=0
$$

that is $T\left(\omega, \xi^{*}(\omega)\right)=\xi^{*}(\omega)$, which implies that $\xi^{*}(\omega)$ is a PPF dependent random fixed point of $T$ on $E_{0}$.

(b) By bart (a) above, the sequence $\left\{\xi_{n}(\omega)\right\}$ of measurable functions as constructed in (a) converges to a random fixed point $\xi^{*}(\omega)$ with PPF dependence. Since $\Re_{c}$ is topologically closed, then $\xi^{*}(\omega) \in \Re_{c}$. Consider $\eta^{*}(\omega) \neq \xi^{*}(\omega)$ be another PPF dependent random fixed point of $T$ for all $\omega \in \Omega$. Then

$$
\begin{aligned}
\left\|\xi^{*}(\omega)-\eta^{*}(\omega)\right\|_{E_{\circ}} & =\left\|\xi^{*}(c, \omega)-\eta^{*}(c, \omega)\right\|_{E}=\left\|T\left(\omega, \xi^{*}(\omega)\right)-T\left(\omega, \eta^{*}(\omega)\right)\right\|_{E} \\
& \leq \beta\left(\left\|\xi^{*}(\omega)-\eta^{*}(\omega)\right\|_{E_{\circ}}\right) M\left(\xi^{*}(\omega), \eta^{*}(\omega)\right)+\gamma\left(\left\|\xi^{*}(\omega)-\eta^{*}(\omega)\right\|_{E_{\circ}}\right) N\left(\xi^{*}(\omega), \eta^{*}(\omega)\right),
\end{aligned}
$$


where

$$
\begin{aligned}
M\left(\xi^{*}(\omega), \eta^{*}(\omega)\right) & =\max \left\{\left\|\xi^{*}(\omega)-\eta^{*}(\omega)\right\|_{E_{\circ}}, \frac{\left\|\xi^{*}(c, \omega)-T\left(\omega, \xi^{*}(\omega)\right)\right\|_{E}\left\|\eta^{*}(c, \omega)-T\left(\omega, \eta^{*}(\omega)\right)\right\|_{E}}{1+\left\|T\left(\omega, \xi^{*}(\omega)\right)-T\left(\omega, \eta^{*}(\omega)\right)\right\|_{E}}\right\} \\
& =\max \left\{\left\|\xi^{*}(\omega)-\eta^{*}(\omega)\right\|_{E_{\circ}}, 0\right\}=\left\|\xi^{*}(\omega)-\eta^{*}(\omega)\right\|_{E_{\circ}},
\end{aligned}
$$

and $N\left(\xi^{*}(\omega), \eta^{*}(\omega)\right)=0$, hence

$$
\left\|\xi^{*}(\omega)-\eta^{*}(\omega)\right\|_{E_{\circ}} \leq \beta\left(\left\|\xi^{*}(\omega)-\eta^{*}(\omega)\right\|_{E_{\circ}}\right)\left\|\xi^{*}(\omega)-\eta^{*}(\omega)\right\|_{E_{\circ}}<\left\|\xi^{*}(\omega)-\eta^{*}(\omega)\right\|_{E_{\circ}}
$$

which is a contradiction, therefore $\xi^{*}(\omega)=\eta^{*}(\omega)$ for all $\omega \in \Omega$, this mean that the random mapping $T$ has a unique PPF dependent fixed point in $\Re_{c} \subseteq E_{\circ}$.

On taking $\beta(t)=r(\omega)$ and $\gamma(t)=\lambda(\omega)$, where $r(\omega)$ and $\lambda(\omega)$ are measurable functions satisfying $0 \leq r(\omega)<1$ and $\lambda(\omega) \geq 0$ in Theorem ??, we obtain the following result:

\section{Corollary 1}

Let $T: \Omega \times E_{\circ} \rightarrow E$ be non-self random mapping such that

$$
\|T(\omega, \xi)-T(\omega, \eta)\|_{E} \leq r(\omega) M(\xi(\omega), \eta(\omega))+\lambda(\omega) N(\xi(\omega), \eta(\omega)),
$$

for all $\xi, \eta \in E_{\circ}, c \in I$ and $\omega \in \Omega$, where

$$
M(\xi(\omega), \eta(\omega))=\max \left\{\|\xi(\omega)-\eta(\omega)\|_{E_{\circ}}, \frac{\|\xi(c, \omega)-T(\omega, \xi(\omega))\|_{E}\|\eta(c, \omega)-T(\omega, \eta(\omega))\|_{E}}{1+\|T(\omega, \xi(\omega))-T(\omega, \eta(\omega))\|_{E}}\right\},
$$

and

$$
N(\xi(\omega), \eta(\omega))=\min \left\{\begin{array}{c}
\|\xi(c, \omega)-T(\omega, \xi(\omega))\|_{E},\|\eta(c, \omega)-T(\omega, \eta(\omega))\|_{E} \\
\|\xi(c, \omega)-T(\omega, \eta(\omega))\|_{E},\|\eta(c, \omega)-T(\omega, \xi(\omega))\|_{E}
\end{array}\right\}
$$

Suppose that $\Re_{c}$ is topologically closed and algebraically closed with respect to the difference, then $T$ has a unique PPF dependent random fixed point $\xi^{*}(\omega) \in \Re_{c}$.

If we take $\lambda(\omega)=0$ in Corollary 1, we get the following corollary:

Corollary 2

Let $T: \Omega \times E_{\circ} \rightarrow E$ be non-self random mapping such that

$$
\|T(\omega, \xi)-T(\omega, \eta)\|_{E} \leq r(\omega) \max \left\{\|\xi(\omega)-\eta(\omega)\|_{E_{\circ}}, \frac{\|\xi(c, \omega)-T(\omega, \xi(\omega))\|_{E}\|\eta(c, \omega)-T(\omega, \eta(\omega))\|_{E}}{1+\|T(\omega, \xi(\omega))-T(\omega, \eta(\omega))\|_{E}}\right\},
$$

for all $\xi, \eta \in E_{\circ}, c \in I$ and $\omega \in \Omega$. If $\Re_{c}$ is topologically and algebraically closed with respect to the difference, then $T$ has a unique PPF dependent random fixed point $\xi^{*}(\omega) \in \Re_{c}$.

Corollary 3

Let $T: \Omega \times E_{\circ} \rightarrow E$ be non-self random mapping such that

$$
\|T(\omega, \xi)-T(\omega, \eta)\|_{E} \leq a(\omega)\|\xi(\omega)-\eta(\omega)\|_{E_{\circ}}+b(\omega) \frac{\|\xi(c, \omega)-T(\omega, \xi(\omega))\|_{E}\|\eta(c, \omega)-T(\omega, \eta(\omega))\|_{E}}{1+\|T(\omega, \xi(\omega))-T(\omega, \eta(\omega))\|_{E}}
$$

for all $\xi, \eta \in E_{\circ}$, where $a(\omega)$ and $b(\omega)$ are measurable functions satisfying $a(\omega), b(\omega) \geq 0$ and $0 \leq a(\omega)+b(\omega)<$ 1 and $c \in I$. Let $\Re_{c}$ is topologically and algebraically closed with respect to the difference, then $T$ has a unique PPF dependent random fixed point $\xi^{*}(\omega) \in \Re_{c}$. Moreover for a fixed $\xi_{\circ}(\omega) \in E_{\circ}$, if the measurable sequence $\left\{\xi_{n}(\omega)\right\}$ of iterates of $T$ be defined by $T\left(\omega, \xi_{n-1}(\omega)\right)=\xi_{n}(c, \omega)$ for all $n \in \mathbb{N}$ and $\omega \in \Omega$. Therefore $\left\{\xi_{n}(\omega)\right\}$ converges to a PPF dependent random fixed point of $T$. 
Proof

Since

$$
\begin{aligned}
& a(\omega)\|\xi(\omega)-\eta(\omega)\|_{E_{\circ}}+b(\omega) \frac{\|\xi(c, \omega)-T(\omega, \xi(\omega))\|_{E}\|\eta(c, \omega)-T(\omega, \eta(\omega))\|_{E}}{1+\|T(\omega, \xi(\omega))-T(\omega, \eta(\omega))\|_{E}} \\
\leq & (a(\omega)+b(\omega)) \max \left\{\|\xi(\omega)-\eta(\omega)\|_{E_{\circ}}, \frac{\|\xi(c, \omega)-T(\omega, \xi(\omega))\|_{E}\|\eta(c, \omega)-T(\omega, \eta(\omega))\|_{E}}{1+\|T(\omega, \xi(\omega))-T(\omega, \eta(\omega))\|_{E}}\right\} .
\end{aligned}
$$

Then from (14), we have

$$
\|T(\omega, \xi)-T(\omega, \eta)\|_{E} \leq r(\omega) \max \left\{\|\xi(\omega)-\eta(\omega)\|_{E_{\circ}}, \frac{\|\xi(c, \omega)-T(\omega, \xi(\omega))\|_{E}\|\eta(c, \omega)-T(\omega, \eta(\omega))\|_{E}}{1+\|T(\omega, \xi(\omega))-T(\omega, \eta(\omega))\|_{E}}\right\}
$$

where $r(\omega)=a(\omega)+b(\omega)$, hence by Corollary 2, we obtain the required.

Now, we prove the existence of PPF dependent random fixed point for random mapping satisfying SuzukiEdelstein type theorem for nonlinear random contractions in Razumikhin class.

Let $\Psi$ be the set of all functions $\psi$ where $\psi:[0,+\infty) \rightarrow[0,+\infty)$ is a continuous nondecreasing function with $\psi(0)=0$ and $\psi(t)<t$ for all $t>0$ such that $\sum_{n=1}^{\infty} \psi^{n}(t)<+\infty$ where $\psi^{n}$ is $\mathbf{n}$-th iterate of $\psi$.

\section{Theorem 3}

Let $(\Omega, X)$ be a measurable space, $E$ be a separable Banach space and $T: \Omega \times E_{\circ} \rightarrow E$ is random operator. Suppose that there exists $\psi \in \Psi$ such that

$$
\frac{1}{2}\|\xi(c, \omega)-T(\omega, \xi(\omega))\|_{E} \leq\|\xi(\omega)-\eta(\omega)\|_{E_{\circ}} \Rightarrow\|T(\omega, \xi(\omega))-T(\omega, \eta(\omega))\|_{E} \leq \psi\left(\|\xi(\omega)-\eta(\omega)\|_{E_{\circ}}\right),
$$

for all $\xi, \eta \in E_{\circ}$ and for some $c \in I$. Assume that $\Re_{c}$ is topologically and algebraically closed with respect to the difference, then $T$ has a unique PPF dependent random fixed point $\xi^{*}(\omega) \in \Re_{c}$. Moreover for a fixed $\xi_{\circ}(\omega) \in E_{\circ}$, if the measurable sequence $\left\{\xi_{n}(\omega)\right\}$ of iterates of $T$ be defined by $T\left(\omega, \xi_{n-1}(\omega)\right)=\xi_{n}(c, \omega)$ for all $n \in \mathbb{N}$ and $\omega \in \Omega$. Therefore $\left\{\xi_{n}(\omega)\right\}$ converges to a PPF dependent random fixed point of $T$.

Proof

Let $\xi_{\circ} \in E_{\circ}$ be arbitrary, by hypothesis $T\left(\omega, \xi_{\circ}(\omega)\right) \in E$, there exists $x_{1}(\omega)=T\left(\omega, \xi_{\circ}(\omega)\right)$, where the function $x_{1}: \Omega \rightarrow E$ is measurable. Choose a measurable function $\xi_{1}: \Omega \rightarrow E_{\circ}$ such that $x_{1}(\omega)=\xi_{1}(c, \omega)$. Continuing this process, by induction, we get

$$
T\left(\omega, \xi_{n-1}(\omega)\right)=\xi_{n}(c, \omega) \text { for all } n \in \mathbb{N} .
$$

If there exists $n_{\circ} \in \mathbb{N}$ such that $\xi_{n_{\circ}}(\omega)=\xi_{n_{\circ}+1}(\omega)$, then $\xi_{n_{\circ}}(c, \omega)=T\left(\omega, \xi_{n_{\circ}}(\omega)\right)$, so there is no thing for prove. Hence let $\left\|\xi_{n-1}(\omega)-\xi_{n}(\omega)\right\|_{E_{\circ}}>0$. Since $\Re_{c}$ is algebraically closed with respect to the difference, then

$$
\left\|\xi_{n-1}(\omega)-\xi_{n}(\omega)\right\|_{E_{\circ}}=\left\|\xi_{n-1}(c, \omega)-\xi_{n}(c, \omega)\right\|_{E},
$$

for all $n \in \mathbb{N}, \omega \in \Omega$ and for some $c \in I$.

Now, we have

$$
\begin{aligned}
\frac{1}{2}\left\|\xi_{n-1}(c, \omega)-T\left(\omega, \xi_{n-1}(\omega)\right)\right\|_{E} & =\frac{1}{2}\left\|\xi_{n-1}(c, \omega)-\xi_{n}(c, \omega)\right\|_{E} \\
& =\frac{1}{2}\left\|\xi_{n-1}(\omega)-\xi_{n}(\omega)\right\|_{E_{\circ}}<\left\|\xi_{n-1}(\omega)-\xi_{n}(\omega)\right\|_{E_{\circ}}
\end{aligned}
$$

So by (15) and (16) we obtain

$$
\begin{aligned}
\left\|\xi_{n}(\omega)-\xi_{n+1}(\omega)\right\|_{E_{\circ}} & =\left\|\xi_{n}(c, \omega)-\xi_{n+1}(c, \omega)\right\|_{E}=\left\|T\left(\omega, \xi_{n-1}(\omega)\right)-T\left(\omega, \xi_{n}(\omega)\right)\right\|_{E} \\
& \leq \psi\left(\left\|\xi_{n-1}(\omega)-\xi_{n}(\omega)\right\|_{E_{\circ}}\right),
\end{aligned}
$$


therefore

$$
\left\|\xi_{n}(\omega)-\xi_{n+1}(\omega)\right\|_{E_{\circ}} \leq \psi^{n}\left(\left\|\xi_{\circ}(\omega)-\xi_{1}(\omega)\right\|_{E_{\circ}}\right) .
$$

For fixed $\epsilon>0$, there exists $N \in \mathbb{N}$ such that

$$
\sum_{n \geq N} \psi^{n}\left(\left\|\xi_{\circ}(\omega)-\xi_{1}(\omega)\right\|_{E_{\circ}}\right)<\epsilon
$$

Let $m, n \in \mathbb{N}$ with $m>n \geq N$, then by triangle inequality, we can write

$$
\left\|\xi_{n}(\omega)-\xi_{m}(\omega)\right\|_{E_{\circ}} \leq \sum_{l=n}^{m-1}\left\|\xi_{l}(\omega)-\xi_{l+1}(\omega)\right\|_{E_{\circ}} \leq \sum_{n \geq N} \psi^{n}\left(\left\|\xi_{\circ}(\omega)-\xi_{1}(\omega)\right\|_{E_{\circ}}\right)<\epsilon .
$$

Consequently, $\lim _{n, m \rightarrow \infty}\left\|\xi_{n}(\omega)-\xi_{m}(\omega)\right\|_{E_{\circ}}=0$. Then $\left\{\xi_{n}(\omega)\right\}$ is a Cauchy sequence of measurable functions on $\Omega$ to $E_{\circ}$. Completeness of $E_{\circ}$ yields that $\left\{\xi_{n}(\omega)\right\}$ converges to measurable function $\xi^{*}: \Omega \rightarrow E_{\circ}$ such that $\lim _{n \rightarrow \infty} \xi_{n}(\omega)=\xi^{*}(\omega)$ for all $\omega \in \Omega$. Since $\Re_{c}$ is topologically closed, we deduce that $\xi^{*}(\omega) \in \Re_{c}$, hence from (17) we have

$$
\left\|\xi_{n}(\omega)-\xi_{n+1}(\omega)\right\|_{E_{\circ}} \leq \psi\left(\left\|\xi_{n-1}(\omega)-\xi_{n}(\omega)\right\|_{E_{\circ}}\right)<\left\|\xi_{n-1}(\omega)-\xi_{n}(\omega)\right\|_{E_{\circ}} .
$$

Suppose that $n_{\circ} \in \mathbb{N}$ such that

$$
\frac{1}{2}\left\|\xi_{n_{\circ}}(c, \omega)-T\left(\omega, \xi_{n_{\circ}}(\omega)\right)\right\|_{E}>\left\|\xi_{n_{\circ}-1}(\omega)-\xi^{*}(\omega)\right\|_{E_{\circ}},
$$

and

$$
\frac{1}{2}\left\|\xi_{n_{\circ}+1}(c, \omega)-T\left(\omega, \xi_{n_{\circ}+1}(\omega)\right)\right\|_{E}>\left\|\xi_{n_{\circ}}(\omega)-\xi^{*}(\omega)\right\|_{E \circ} .
$$

Then from (18) and triangle inequality, we obtain

$$
\begin{aligned}
\left\|\xi_{n_{\circ}-1}(\omega)-\xi_{n_{\circ}}(\omega)\right\|_{E_{\circ}} & \leq\left\|\xi_{n_{\circ}-1}(\omega)-\xi^{*}(\omega)\right\|_{E_{\circ}}+\left\|\xi_{n_{\circ}}(\omega)-\xi^{*}(\omega)\right\|_{E_{\circ}} \\
& <\frac{1}{2}\left\|\xi_{n_{\circ}}(c, \omega)-T\left(\omega, \xi_{n_{\circ}}(\omega)\right)\right\|_{E}+\frac{1}{2}\left\|\xi_{n_{\circ}+1}(c, \omega)-T\left(\omega, \xi_{n_{\circ}+1}(\omega)\right)\right\|_{E} \\
& =\frac{1}{2}\left\|\xi_{n_{\circ}}(c, \omega)-\xi_{n_{\circ}+1}(c, \omega)\right\|_{E}+\frac{1}{2}\left\|\xi_{n_{\circ}+1}(c, \omega)-\xi_{n_{\circ}+2}(c, \omega)\right\|_{E} \\
& =\frac{1}{2}\left\|\xi_{n_{\circ}}(\omega)-\xi_{n_{\circ}+1}(\omega)\right\|_{E_{\circ}}+\frac{1}{2}\left\|\xi_{n_{\circ}+1}(\omega)-\xi_{n_{\circ}+2}(\omega)\right\|_{E_{\circ}} \\
& \leq \frac{1}{2}\left\|\xi_{n_{\circ}}(\omega)-\xi_{n_{\circ}-1}(\omega)\right\|_{E_{\circ}}+\frac{1}{2}\left\|\xi_{n_{\circ}}(\omega)-\xi_{n_{\circ}-1}(\omega)\right\|_{E_{\circ}} \\
& =\left\|\xi_{n_{\circ}}(\omega)-\xi_{n_{\circ}-1}(\omega)\right\|_{E_{\circ}},
\end{aligned}
$$

which is a contradiction, therefore either,

$$
\frac{1}{2}\left\|\xi_{n}(c, \omega)-T\left(\omega, \xi_{n}(\omega)\right)\right\|_{E} \leq\left\|\xi_{n-1}(\omega)-\xi^{*}(\omega)\right\|_{E_{\circ}}
$$

or

$$
\frac{1}{2}\left\|\xi_{n+1}(c, \omega)-T\left(\omega, \xi_{n+1}(\omega)\right)\right\|_{E} \leq\left\|\xi_{n}(\omega)-\xi^{*}(\omega)\right\|_{E_{\circ}},
$$

holds for all $n \in \mathbb{N}, \omega \in \Omega$ and for some $c \in I$. Consider (19) is hold, then (15) yields that

$$
\begin{aligned}
\left\|T\left(\omega, \xi^{*}(\omega)\right)-\xi^{*}(c, \omega)\right\|_{E} & \leq\left\|T\left(\omega, \xi^{*}(\omega)\right)-T\left(\omega, \xi_{n}(\omega)\right)\right\|_{E}+\left\|T\left(\omega, \xi_{n}(\omega)\right)-\xi^{*}(c, \omega)\right\|_{E} \\
& =\left\|T\left(\omega, \xi^{*}(\omega)\right)-T\left(\omega, \xi_{n}(\omega)\right)\right\|_{E}+\left\|\xi_{n+1}(c, \omega)-\xi^{*}(c, \omega)\right\|_{E} \\
& \leq \psi\left(\left\|\xi^{*}(\omega)-\xi_{n}(\omega)\right\|_{E_{\circ}}\right)+\left\|\xi_{n+1}(\omega)-\xi^{*}(\omega)\right\|_{E_{\circ}} .
\end{aligned}
$$


Taking limit as $n \rightarrow \infty$ in the above inequality, we have $\left\|T\left(\omega, \xi^{*}(\omega)\right)-\xi^{*}(c, \omega)\right\|_{E}=0$, i.e.,

$$
T\left(\omega, \xi^{*}(\omega)\right)=\xi^{*}(c, \omega) .
$$

By a similar way, we obtain (21) if (20) holds for all $n \in \mathbb{N}$. This implies that $\xi^{*}(\omega)$ is a PPF dependent random fixed point of $T$.

For uniqueness, suppose that $\eta^{*}(\omega) \neq \xi^{*}(\omega)$ be another PPF dependent random fixed point of $T$ for all $\omega \in \Omega$. So

$$
\frac{1}{2}\left\|\xi^{*}(c, \omega)-T\left(\omega, \xi^{*}(\omega)\right)\right\|_{E}=0 \leq\left\|\xi^{*}(\omega)-\eta^{*}(\omega)\right\|_{E_{\circ}},
$$

hence from (15), we can write

$$
\begin{aligned}
\left\|\xi^{*}(\omega)-\eta^{*}(\omega)\right\|_{E_{\circ}} & =\left\|\xi^{*}(c, \omega)-\eta^{*}(c, \omega)\right\|_{E}=\left\|T\left(\omega, \xi^{*}(\omega)\right)-T\left(\omega, \eta^{*}(\omega)\right)\right\|_{E} \\
& \leq \psi\left(\left\|\xi^{*}(\omega)-\eta^{*}(\omega)\right\|_{E_{\circ}}\right)<\left\|\xi^{*}(\omega)-\eta^{*}(\omega)\right\|_{E_{\circ}},
\end{aligned}
$$

a contradiction again. Hence $\eta^{*}(\omega)=\xi^{*}(\omega)$.

If we take $\psi(t)=r(\omega)$ (where $r(\omega)$ as in Corollary 1 in Theorem 3, we obtain the following result:

Corollary 4

Let $T: \Omega \times E_{\circ} \rightarrow E$ be non-self random mapping. Suppose there exists $0 \leq r(\omega)<1$ such that

$$
\begin{aligned}
\frac{1}{2}\|\xi(c, \omega)-T(\omega, \xi(\omega))\|_{E} & \leq\|\xi(\omega)-\eta(\omega)\|_{E_{\circ}} \\
& \Rightarrow\|T(\omega, \xi(\omega))-T(\omega, \eta(\omega))\|_{E} \leq r(\omega)\|\xi(\omega)-\eta(\omega)\|_{E_{\circ}},
\end{aligned}
$$

for all $\xi, \eta \in E_{\circ}$. Assume $\Re_{c}$ is topologically and algebraically closed with respect to the difference, then $T$ has a unique PPF dependent random fixed point $\xi^{*}(\omega) \in \Re_{c}$. Moreover for a fixed $\xi_{\circ}(\omega) \in E_{\circ}$, if the measurable sequence $\left\{\xi_{n}(\omega)\right\}$ of iterates of $T$ be defined by $T\left(\omega, \xi_{n-1}(\omega)\right)=\xi_{n}(c, \omega)$ for all $n \in \mathbb{N}$ and $\omega \in \Omega$. Then $\left\{\xi_{n}(\omega)\right\}$ converges to a PPF dependent random fixed point of $T$.

\section{Random coincidence point with PPF dependence}

We begin this section with the following definitions:

Let $T: \Omega \times E_{\circ} \rightarrow E$ and $S: \Omega \times E_{\circ} \rightarrow E_{\circ}$ be two random operators. A point $\xi^{*}(\omega) \in E_{\circ}$ is called a PPF dependent random coincidence point of $T$ and if

$$
T\left(\omega, \xi^{*}(\omega)\right)=S\left(\omega, \xi^{*}(c, \omega)\right),
$$

for some $c \in I$ and $\omega \in \Omega$.

The random operators $T: \Omega \times E_{\circ} \rightarrow E$ and $S: \Omega \times E_{\circ} \rightarrow E_{\circ}$ are said to satisfy a condition of hybrid rational Geraghty random contraction if there exists $\beta \in \digamma$ and $c \in I$ such that

$$
\begin{aligned}
\|T(\omega, \xi)-T(\omega, \eta)\|_{E} \leq & \beta\left(\|S(\omega, \xi(\omega))-S(\omega, \eta(\omega))\|_{E_{\circ}}\right) M^{S}(\xi(\omega), \eta(\omega)) \\
& +\gamma\left(\|S(\omega, \xi(\omega))-S(\omega, \eta(\omega))\|_{E_{\circ}}\right) N^{S}(\xi(\omega), \eta(\omega)),
\end{aligned}
$$

for all $\xi, \eta \in E_{\circ}$, where $\gamma:[0, \infty) \rightarrow[0, \infty)$ is a bounded function,

$$
M^{S}(\xi(\omega), \eta(\omega))=\max \left\{\begin{array}{c}
\|S(\omega, \xi(\omega))-S(\omega, \eta(\omega))\|_{E_{\odot}} \\
\frac{\|S(\omega, \xi(c, \omega))-T(\omega, \xi(\omega))\|_{E}\|S(\omega, \eta(c, \omega))-T(\omega, \eta(\omega))\|_{E}}{1+\|T(\omega, \xi(\omega))-T(\omega, \eta(\omega))\|_{E}}
\end{array}\right\}
$$

and

for all $\omega \in \Omega$.

$$
N^{S}(\xi(\omega), \eta(\omega))=\min \left\{\begin{array}{c}
\|S(\omega, \xi(c, \omega))-T(\omega, \xi(\omega))\|_{E},\|S(\omega, \eta(c, \omega))-T(\omega, \eta(\omega))\|_{E}, \\
\|S(\omega, \xi(c, \omega))-T(\omega, \eta(\omega))\|_{E},\|S(\omega, \eta(c, \omega))-T(\omega, \xi(\omega))\|_{E}
\end{array}\right\},
$$

Now, according to Theorem 2, we state and prove the following PPF dependent random coincidence theorem. 


\section{Theorem 4}

Let $(\Omega, X)$ be a measurable space, $E$ be a separable Banach space and $T: \Omega \times E_{\circ} \rightarrow E$ and $S: \Omega \times E_{\circ} \rightarrow E_{\circ}$ satisfy a condition of hybrid rational Geraghty random contraction (22) such that $S\left(\Re_{c}\right) \subseteq \Re_{c}$. Consider $S\left(\Re_{c}\right)$ is topologically and algebraically closed with respect to the difference. Then $T$ and $S$ have a PPF dependent random coincidence point in $\Re_{c}$.

\section{Proof}

The idea of the proof be defined as a new random mapping satisfy all conditions of Theorem 2 as follows. Since $S: \Omega \times E_{\circ} \rightarrow E_{\circ}$, so there exists a measurable function $C_{\circ}(\omega) \in E_{\circ}$ such that $S\left(\omega, C_{\circ}\right)=S\left(\omega, E_{\circ}\right)$ and $S$ is one-one. As $T\left(\omega, C_{\circ}\right) \subseteq S\left(\omega, E_{\circ}\right) \subseteq E$, therefore we can define a random mapping $F: \Omega \times S \rightarrow E$ (note that $\left.S=S\left(\omega, C_{\circ}\right)\right)$ by $F(\omega, S(\omega, \xi))=T(\omega, \zeta(\omega))$, for all $\zeta \in C_{\circ}$ and $\omega \in \Omega$. Since $\left.S\right|_{C_{\circ}}$ is one to one, then $F$ welldefined.

Since the pair $(S, T)$ satisfy (22) then, we have

$$
\begin{aligned}
\|F(\omega, S(\omega, \xi))-F(\omega, S(\omega, \eta))\|_{E}= & \|T(\omega, \xi(\omega))-T(\omega, \eta(\omega))\|_{E} \\
\leq & \beta\left(\|S(\omega, \xi(\omega))-S(\omega, \eta(\omega))\|_{E_{\circ}}\right) M^{S}(\xi(\omega), \eta(\omega)) \\
& +\gamma\left(\|S(\omega, \xi(\omega))-S(\omega, \eta(\omega))\|_{E_{\circ}}\right) N^{S}(\xi(\omega), \eta(\omega)),
\end{aligned}
$$

for all $\xi, \eta \in E_{\circ}$ where

$$
M^{S}(\xi(\omega), \eta(\omega))=\max \left\{\begin{array}{c}
\|S(\omega, \xi(\omega))-S(\omega, \eta(\omega))\|_{E_{o}} \\
\frac{\|S(\omega, \xi(c, \omega))-F(\omega, S(\omega, \xi))\|_{E}\|S(\omega, \eta(c, \omega))-F(\omega, S(\omega, \eta))\|_{E}}{1+\|F(\omega, S(\omega, \xi))-F(\omega, S(\omega, \eta))\|_{E}}
\end{array}\right\},
$$

and

$$
N^{S}(\xi(\omega), \eta(\omega))=\min \left\{\begin{array}{c}
\|S(\omega, \xi(c, \omega))-F(\omega, S(\omega, \xi))\|_{E},\|S(\omega, \eta(c, \omega))-F(\omega, S(\omega, \eta))\|_{E}, \\
\|S(\omega, \xi(c, \omega))-F(\omega, S(\omega, \eta))\|_{E},\|S(\omega, \eta(c, \omega))-F(\omega, S(\omega, \xi))\|_{E}
\end{array}\right\}
$$

Which implies that $F$ is a hybrid rational Geraghty random contractive mapping and all conditions of Theorem 2 are satisfied. Then there exists a unique PPF dependent random fixed point $\zeta(\omega) \in S\left(\omega, C_{\circ}\right)$ of $F$, with meaning $F(\omega, \zeta(\omega))=\zeta(c, \omega)$, where $\zeta: \Omega \rightarrow E_{0}$ is measurable function and $c \in I$. Again since $\zeta(\omega) \in S\left(\omega, C_{\circ}\right)$, then there exists a measurable function $\varphi(\omega) \in C_{\circ}$ such that $\zeta(\omega)=S(\omega, \varphi(\omega))$, so

$$
T(\omega, \varphi(\omega))=F(\omega, S(\omega, \varphi))=F(\omega, \zeta(\omega))=\zeta(c, \omega)=S(\omega, \varphi(c, \omega)) .
$$

Hence $\varphi(\omega)$ is a PPF dependent random coincidence point of $S$ and $T$, this complete the proof.

According to the previous corollaries and Theorem 4, we can obtain the following results:

\section{Corollary 5}

Let $T: \Omega \times E_{\circ} \rightarrow E$ and $S: \Omega \times E_{\circ} \rightarrow E_{\circ}$ be two random mappings, there exists $c \in I$ such that $S\left(\Re_{c}\right) \subseteq \Re_{c}$ and

$$
\|T(\omega, \xi)-T(\omega, \eta)\|_{E} \leq r(\omega) M^{S}(\xi(\omega), \eta(\omega))+\lambda(\omega) N^{S}(\xi(\omega), \eta(\omega)),
$$

for all $\xi, \eta \in E_{\circ}$ and $\omega \in \Omega,(r(\omega), \lambda(\omega)$ as in Corollary 1 where

$$
M^{S}(\xi(\omega), \eta(\omega))=\max \left\{\begin{array}{c}
\|S(\omega, \xi(\omega))-S(\omega, \eta(\omega))\|_{E_{\circ}} \\
\frac{\|S(\omega, \xi(c, \omega))-T(\omega, \xi(\omega))\|_{E}\|S(\omega, \eta(c, \omega))-T(\omega, \eta(\omega))\|_{E}}{1+\|T(\omega, \xi(\omega))-T(\omega, \eta(\omega))\|_{E}}
\end{array}\right\}
$$

and

$$
N^{S}(\xi(\omega), \eta(\omega))=\min \left\{\begin{array}{c}
\|S(\omega, \xi(c, \omega))-T(\omega, \xi(\omega))\|_{E},\|S(\omega, \eta(c, \omega))-T(\omega, \eta(\omega))\|_{E}, \\
\|S(\omega, \xi(c, \omega))-T(\omega, \eta(\omega))\|_{E},\|S(\omega, \eta(c, \omega))-T(\omega, \xi(\omega))\|_{E}
\end{array}\right\},
$$

Let $S\left(\Re_{c}\right)$ is topologically and algebraically closed with respect to the difference. Then $T$ and $S$ have a PPF dependent random coincidence point. 
Corollary 6

Let $T: \Omega \times E_{\circ} \rightarrow E$ and $S: \Omega \times E_{\circ} \rightarrow E_{\circ}$ be two random mappings, there exists $c \in I$ such that $S\left(\Re_{c}\right) \subseteq \Re_{c}$ and

$$
\|T(\omega, \xi)-T(\omega, \eta)\|_{E} \leq r(\omega) \max \left\{\begin{array}{c}
\|S(\omega, \xi(\omega))-S(\omega, \eta(\omega))\|_{E_{o}} \\
\frac{\|S(\omega, \xi(c, \omega))-T(\omega, \xi(\omega))\|_{E}\|S(\omega, \eta(c, \omega))-T(\omega, \eta(\omega))\|_{E}}{1+\|T(\omega, \xi(\omega))-T(\omega, \eta(\omega))\|_{E}}
\end{array}\right\}
$$

for all $\xi, \eta \in E_{\circ}, 0 \leq r(\omega)<1$. Suppose that $S\left(\Re_{c}\right)$ is topologically and algebraically closed with respect to the difference. Then $T$ and $S$ have a PPF dependent random coincidence point.

Corollary 7

Let $T: \Omega \times E_{\circ} \rightarrow E$ and $S: \Omega \times E_{\circ} \rightarrow E_{\circ}$ be two random mappings, there exists $c \in I$ such that $S\left(\Re_{c}\right) \subseteq \Re_{c}$ and

$$
\begin{aligned}
\|T(\omega, \xi)-T(\omega, \eta)\|_{E} \leq & a(\omega)\|S(\omega, \xi(\omega))-S(\omega, \eta(\omega))\|_{E_{\circ}} \\
& +b(\omega) \frac{\|S(\omega, \xi(c, \omega))-T(\omega, \xi(\omega))\|_{E}\|S(\omega, \eta(c, \omega))-T(\omega, \eta(\omega))\|_{E}}{1+\|T(\omega, \xi(\omega))-T(\omega, \eta(\omega))\|_{E}},
\end{aligned}
$$

for all $\xi, \eta \in E_{\circ}$ and $a(\omega), b(\omega)$ as in Corollary 3. Let $S\left(\Re_{c}\right)$ is topologically and algebraically closed with respect to the difference. Then $T$ and $S$ have a PPF dependent random coincidence point.

\section{Corollary 8}

Let $(\Omega, X)$ be a measurable space, $E$ be a separable Banach space, $T: \Omega \times E_{\circ} \rightarrow E$ and $S: \Omega \times E_{\circ} \rightarrow E_{\circ}$ be two random mappings such that $S\left(\Re_{c}\right) \subseteq \Re_{c}$ and

$$
\begin{aligned}
\frac{1}{2}\|S(\omega, \xi(c, \omega))-T(\omega, \xi(\omega))\|_{E} & \leq\|S(\omega, \xi(\omega))-S(\omega, \eta(\omega))\|_{E_{\circ}} \\
& \Rightarrow\|T(\omega, \xi(\omega))-T(\omega, \eta(\omega))\|_{E} \leq \psi\left(\|S(\omega, \xi(\omega))-S(\omega, \eta(\omega))\|_{E_{\circ}}\right),
\end{aligned}
$$

for all $\xi, \eta \in E_{\circ}$, where $\psi \in \Psi$. Let $S\left(\Re_{c}\right)$ is topologically and algebraically closed with respect to the difference. Then $T$ and $S$ have a PPF dependent random coincidence point.

\section{Corollary 9}

Let $T: \Omega \times E_{\circ} \rightarrow E$ and $S: \Omega \times E_{\circ} \rightarrow E_{\circ}$ be two random mappings such that $S\left(\Re_{c}\right) \subseteq \Re_{c}$ and

$$
\begin{aligned}
\frac{1}{2}\|S(\omega, \xi(c, \omega))-T(\omega, \xi(\omega))\|_{E} & \leq\|S(\omega, \xi(\omega))-S(\omega, \eta(\omega))\|_{E_{\circ}} \\
& \Rightarrow\|T(\omega, \xi(\omega))-T(\omega, \eta(\omega))\|_{E} \leq r(\omega)\|S(\omega, \xi(\omega))-S(\omega, \eta(\omega))\|_{E_{\circ}},
\end{aligned}
$$

for all $\xi, \eta \in E_{\circ}$, where $0 \leq r(\omega)<1$. Let $S\left(\Re_{c}\right)$ is topologically and algebraically closed with respect to the difference. Then $T$ and $S$ have a PPF dependent random coincidence point.

Note that the random operator $T$ in Theorems 2-3 and Corollaries 1-4 and the random pair $(S, T)$ in Theorems 4-8 and Corollaries 5-9 are not required to satisfy any continuity condition on the domains of their definition.

\section{Application to random differential and integral equations}

Fixed point theorems have many applications in many mathematical disciplines especially in differential and integral equations (see [1, 13, 15, 20]. In this section, we shall prove the existence of PPF dependence solution to a periodic boundary random-valued problem type (in short PBRVP).

Let $I_{\circ}=[-t, 0]$ and $I=[0, T]$ are two closed and bounded intervals in $\mathbb{R}$, for reals $t, T>0$ and let $\aleph$ denote the space of continuous real-valued functions defined on $I_{\circ}$. We define a distance with the supermum norm $\|\cdot\|_{\aleph}$ by

$$
\|\xi\|_{\aleph}=\sup _{s \in I_{\circ}}\left|\xi_{s}\right| \text {. }
$$


It's clear that $\aleph$ is a Banach space with this norm. For $t \in I$ define a function $t \rightarrow x_{t} \in \aleph$ by

$$
x_{t}(s)=x(t+s), s \in I_{\circ},
$$

where the argument $s$ represents the delay in the argument solution.

Let $(\Omega, X)$ be a measurable space. Define a mapping $x: \Omega \rightarrow C(J, \mathbb{R})$, we denote a function $x(t, \omega)$, which is continuous in the variable $\mathrm{t}$ for each $\omega \in \Omega$, we also write $x(t, \omega)=x(\omega)(t)$. Given the measurable functions $\varphi: \Omega \rightarrow \aleph$ and $x: \Omega \rightarrow C(I, \mathbb{R})$, consider the first-order periodic boundary random-valued problem (PBRVP)

$$
\left\{\begin{array}{c}
x^{\prime}(t, \omega)=f\left(t, x(t, \omega), x_{t}(\omega), \omega\right), \\
x_{\circ}(\omega)=\phi_{\circ}(\omega) \\
x(0, \omega)=x(T, \omega)=\phi_{\circ}(0, \omega)
\end{array}\right.
$$

for all $t \in I$ and $\omega \in \Omega$, where $f: I \times \mathbb{R} \times \aleph \rightarrow \mathbb{R}$. By a random solution $x$ of PBRVP (23) we mean a measurable function $x: \Omega \rightarrow C(J, \mathbb{R})$ that satisfies the equation (23) on $J$, where $C(J, \mathbb{R})$ is the space of continuous real-valued functions defined on $J=I \cup I_{\circ}$.

In this fashion, we will prove the existence of random solutions with PPF dependence for the PBRVP (23) defined on $J$ with the condition of Theorem 3 . We consider the following hypotheses:

$\left(\mathrm{H}_{1}\right)$ The function $\omega \rightarrow f(t, x, \omega)$ is measurable for each $t \in I$ and $x \in \aleph$ and the function $(t, x) \rightarrow f(t, x, \omega)$ is jointly continuous for each $\omega \in \Omega$.

$\left(\mathrm{H}_{2}\right)$ Assume that there exists $\lambda>0$ such that for each $\tilde{x}, \tilde{y}: \Omega \rightarrow C(J, \mathbb{R})$ and $\xi, \eta \in \aleph$ with

$$
\frac{1}{2}\left\|\xi(\omega)-\int_{0}^{T} G(t, s) F(s, x(s, \omega), \xi(\omega), \omega) d s\right\|_{E} \leq\|\xi(\omega)-\eta(\omega)\|_{\aleph},
$$

we have

$$
|[f(t, x(t, \omega), \xi(\omega), \omega)+\lambda x(t, \omega)]-[f(t, x(t, \omega), \eta(\omega), \omega)+\lambda x(t, \omega)]| \leq \lambda \psi\left(\|\xi(\omega)-\eta(\omega)\|_{\aleph}\right) .
$$

Theorem 5

Suppose the conditions $\left(\mathrm{H}_{1}\right)$ and $\left(\mathrm{H}_{2}\right)$ hold, then PBRVP (23) has a unique PPF dependent random solution defined on $J$.

Proof

Suppose that $E=C(J, \mathbb{R})$ which is a separable Banach space. Given a function $x: \Omega \rightarrow C(J, \mathbb{R})$, define a mapping $\tilde{x}: I \rightarrow \aleph$ by $\tilde{x}(t)(0)=x_{t}(0)=x(t), t \in I$ and $\tilde{x}(0)=x_{\circ}$. Define a set $\tilde{E}$ of functions by

$$
\widetilde{E}=\left\{\tilde{x}=\left(x_{t}\right)_{t \in I}: x_{t} \in \aleph, x \in E \text { and } x(0)=x(T)=\phi_{\circ}(0), x_{\circ}=\phi_{\circ}\right\} .
$$

We define a norm on $\widetilde{E}$ by

$$
\|\tilde{x}-\tilde{y}\|_{\tilde{E}}=\sup _{t \in I} \max _{-t \leq s \leq 0}\left|x_{t}(s)-y_{t}(s)\right|=\sup _{t \in I}\left\|x_{t}-y_{t}\right\|_{\aleph} .
$$

Clearly, $\tilde{x}, \tilde{y} \in C\left(I_{\circ}, \mathbb{R}\right)=\aleph$. Now we show that $\tilde{E}$ is a Banach space. Let $\left\{\tilde{x}_{n}\right\}$ be a Cauchy sequence in $\tilde{E}$ and $\tilde{x}_{n}(t)=x_{t}^{n}$, then $\left\{\left(x_{t}^{n}\right)_{t \in I}\right\}$ is a Cauchy sequence in $\aleph$ for each $t \in I$. This implies that $\left\{x_{t}^{m}(s)\right\}$ is a Cauchy sequence in $\mathbb{R}$ for each $s \in I_{\circ}$. Then $\left\{x_{t}^{m}(s)\right\}$ converges to $x_{t}(s)$ for each $t \in I$. Since $\left\{x_{t}^{n}\right\}$ is a sequence of uniformly continuous functions for a fixed $t \in I, x_{t}(s)$ is also continuous in $s \in I_{\circ}$. So the sequence $\left.\tilde{\{} x_{n}\right\}$ converges to $\tilde{x} \in \widetilde{E}$. Since $\widetilde{E}$ is complete, moreover, $\widetilde{E}$ is a separable Banach space.

The PBRVP (23) can be formulated as follows:

$$
\left\{\begin{array}{c}
x^{\prime}(t, \omega)+\lambda x(t, \omega)=f\left(t, x(t, \omega), x_{t}(\omega), \omega\right)+\lambda x(t, \omega), \\
x_{\circ}(\omega)=\phi_{\circ}(\omega) \\
x(0, \omega)=x(T, \omega)=\phi_{\circ}(0, \omega) .
\end{array}\right.
$$

Stat., Optim. Inf. Comput. Vol. 7, December 2019 
Consider

where $t \in I$.

$$
\left\{\begin{array}{c}
x^{\prime}(t, \omega)+\lambda x(t, \omega)=\rho(t, \omega)=F\left(t, x(t, \omega), x_{t}(\omega), \omega\right) \\
x_{\circ}(\omega)=\phi_{\circ}(\omega) \\
x(0, \omega)=x(T, \omega)=\phi_{\circ}(0, \omega)
\end{array}\right.
$$

Using variation of parameters formula, we get

$$
x(t, \omega)=x(0, \omega) e^{-\lambda t}+\int_{0}^{t} e^{-\lambda(t-s)} \rho(s, \omega) d s,
$$

which yields

$$
x(T, \omega)=x(0, \omega) e^{-\lambda T}+\int_{0}^{T} e^{-\lambda(T-s)} \rho(s, \omega) d s .
$$

Since $x(0, \omega)=x(T, \omega)$, we have

$$
x(0, \omega)\left[1-e^{-\lambda T}\right]=e^{-\lambda T} \int_{0}^{T} e^{\lambda s} \rho(s, \omega) d s,
$$

or

$$
x(0, \omega)=\frac{1}{\left[1-e^{-\lambda T}\right]} \int_{0}^{T} e^{\lambda s} \rho(s, \omega) d s .
$$

Applying (25) in (24), we can write

$$
x(t, \omega)=\int_{0}^{T} G(t, s) \rho(s, \omega) d s
$$

where

$$
G(t, s)= \begin{cases}\frac{e^{\lambda(T+s-t)}}{e^{\lambda T}-1}, & 0 \leq s \leq t \leq T \\ \frac{e^{\lambda(s-t)}}{e^{\lambda T}-1}, & 0 \leq t \leq s \leq T\end{cases}
$$

Define the operator $S: \Omega \times \widetilde{E} \rightarrow \mathbb{R}$ as

$$
S(\omega, \tilde{x}(t, \omega))=\int_{0}^{T} G(t, s) F\left(s, x(s, \omega), x_{s}(\omega), \omega\right) d s .
$$

First, we show that $S$ is a random operator on $\Omega \times \widetilde{E}$. Since hypothesis $\left(\mathrm{H}_{1}\right)$ holds, by Caratheodory theorem, the function $\omega \rightarrow f(t, x, \omega)$ is measurable for all $t \in I$ and $x \in \aleph$. As integral is the limit of the finite sum of measurable function, the map

$$
\omega \rightarrow \int_{0}^{T} G(t, s) F\left(s, x(s, \omega), x_{s}(\omega), \omega\right) d s,
$$

is measurable. Hence the operator $S(\omega, \tilde{x})$ is measurable in $\omega$ for each $\tilde{x} \in \tilde{E}$. Thus we have the operator $S$ is random operator on $\Omega \times \tilde{E}$.

Secondly, we claim that the random operator $S$ is continuous on $\tilde{E}$. Let $\omega \in \Omega$ be a fixed Suppose that $\left\{\tilde{x}_{n}(\omega)\right\}$ be a sequence of points in $\tilde{E}$ such that $\widetilde{x}_{n}(\omega) \rightarrow \widetilde{x}(\omega)$ as $n \rightarrow \infty$. Then by dominated convergence theorem, we have

$$
\begin{aligned}
\lim _{n \rightarrow \infty} S\left(\omega, \tilde{x_{n}}(t, \omega)\right) & =\lim _{n \rightarrow \infty}\left(\int_{0}^{T} G(t, s) F\left(s, x(s, \omega), x_{s}^{n}(\omega), \omega\right) d s\right) \\
& =\int_{0}^{T} G(t, s) \lim _{n \rightarrow \infty}\left(F\left(s, x(s, \omega), x_{s}^{n}(\omega), \omega\right)\right) d s \\
& =\int_{0}^{T} G(t, s)\left(F\left(s, x(s, \omega), x_{s}(\omega), \omega\right)\right) d s=S(\omega, \tilde{x}(t, \omega)),
\end{aligned}
$$


for all $t \in I$.

Finally, from condition $\left(\mathrm{H}_{2}\right)$, we get

$$
\begin{aligned}
|S(\omega, \tilde{x}(t, \omega))-S(\omega, \tilde{y}(t, \omega))| & =\int_{0}^{T} G(t, s)\left[F\left(s, x(s, \omega), x_{s}(\omega), \omega\right)-F\left(s, y(s, \omega), y_{s}(\omega), \omega\right)\right] d s \\
\leq & \int_{0}^{T} G(t, s) \alpha \psi\left(\|x(\omega)-y(\omega)\|_{\aleph}\right) d s \\
& \leq \lambda \psi\left(\|\tilde{x}(\omega)-\tilde{y}(\omega)\|_{\tilde{E}}\right)\left[\int_{0}^{t} \frac{e^{\lambda(T+s-t)}}{e^{\lambda T}-1} d s+\int_{0}^{T} \frac{e^{\lambda(s-t)}}{e^{\lambda T}-1} d s\right] \\
& =\lambda \psi\left(\|\tilde{x}(\omega)-\tilde{y}(\omega)\|_{\tilde{E}}\right)\left[\frac{1}{\lambda\left(e^{\lambda T}-1\right)}\left(\left.e^{\lambda(T+s-t)}\right|_{0} ^{t}+\left.e^{\lambda(s-t)}\right|_{t} ^{T}\right)\right] \\
& =\lambda \psi\left(\|\tilde{x}(\omega)-\tilde{y}(\omega)\|_{\tilde{E}}\right)\left[\frac{1}{\lambda\left(e^{\lambda T}-1\right)}\left(e^{\lambda T}-e^{\lambda(T-t)}+e^{\lambda(T-t)}-1\right)\right] \\
& =\psi\left(\|\tilde{x}(\omega)-\tilde{y}(\omega)\|_{\tilde{E}} \cdot\right.
\end{aligned}
$$

Hence, all conditions of Theorem 3 are satisfied and so, there exists a unique random fixed point with a

PPF dependent $\tilde{x^{*}}(t, \omega) \in \tilde{E}$ such that $S\left(\omega, \tilde{x^{*}}(t, \omega)\right)=\left(\tilde{x^{*}}(t, \omega)\right)_{t \in I}$. This yields the functional random integral equation (26) has a random solution with PPF dependence defined on $J$ which implies that the PBRVP (23) has a PPF dependent random solution. Moreover, here the Razumikhin class $\Re_{0}, 0 \in[-r, T]$ is $C([0, T], \mathbb{R})$ which is topologically and algebraically closed with respect to the difference, so this solution is unique.

\section{Conclusion}

In this paper we introduced some random fixed point theorems with PPF (past, present and future) in a separable Banach space under hybrid rational and Suzuki Edelstein random contractions type without using the continuity condition of the mappings. The new approach improves, extends and generalizes the existing results in the literature of the fixed point theory. As an application, we give the existence of random solution to certain nonlinear functional random differentiable equations with a PPF dependent.

\section{REFERENCES}

1. R. P. Agarwal, N. Hussain, and M. A. Taoudi, Fixed point theorems in ordered Banach spaces and applications to nonlinear integral equations, Abstr. Appl. Anal., Art. ID 245872, pp. 1-15, 2012.

2. R. P. Agarwal, P. Kumam, and W. Sintunavarat, PPF dependent fixed point theorems for an $\alpha c$-admissible non-self mapping in the Razumikhin class, Fixed Point Theory and Appl., vol. 280, pp. 1-14, 2013.

3. S. R. Bernfeld, V. Lakshmikantham, and Y. M. Reddy, Fixed point theorems of operators with PPF dependence in Banach spaces, Applicable Anal., Vol. 6, pp. 271-280, 1977.

4. A. T. Bharucha-Reid, Fixed point theorems in probabilistic analysis, Bull. Amer. Math. Soc., vol. 82, No. 5, pp. 641-657, 1976.

5. B. C. Dhage, Fixed point theorems with PPF dependence and functional differential equations, Fixed Point Theory, vol. 13, no. 2, pp. 439-452, 2012.

6. B. C. Dhage, On some common fixed point theorems with PPF dependence in Banach spaces, J. Nonlinear Sci. Appl., vol. 5, no. 3, pp. 220-232, 2012.

7. B. C. Dhage, Some basic random fixed point theorems with PPF dependence and functional random differential equations, Differ. Equ. Appl., vol. 4 no. 2, PP. 181-195, 2012.

8. Z. Drici, F.A. Mcrae, and J. Vasundhara Devic, Fixed-point theorems in partially ordered metric spaces for operators with PPF dependence, Nonlinear Analysis, vol. 67, pp. 641-647, 2017.

9. Z. Drici, F. A. Mcrae, and J. V. Devi, Fixed point theorems for mixed monotone operators with PPF dependence, Nonlinear Anal., vol. 69, no. 2, pp. 632-636, 2008.

10. D. Dukić, Z. Kadelburg, and S. Radenović, Fixed Points of Geraghty-Type Mappings in arious Generalized Metric Spaces, Abstr. Appl. Anal., vol. 2011, pp. 1-13, 2011.

11. M. Geraghty, On contractive mappings, Proc. Amer. Math. Soc., vol. 40, pp. 604-608, 1973. 
12. P. Hans, Random fixed point theorems, Transactions of the first Prague conference on information theory, statistical decision functions, random processes held at Liblice near Prague from November, 28 to 30, pp. 105-125, 1957.

13. N. Hussain, A. R. Khan, and R. P. Agarwal, Krasnoselskii and Ky Fan type fixed point theorems in ordered Banach spaces, J. Nonlinear Convex Anal., vol. 11, no. 3, pp. 475-489, 2010.

14. N. Hussain, S. Khaleghizadeh, P. Salimi, and F. Akbar, New fixed point results with PPF dependence in Banach spaces endowed with a graph, Abstract and Applied Analysis, vol. 2013, pp. 1-9, 2013.

15. N. Hussain, and M. A. Taoudi, Krasnoselskii-type fixed point theorems with applications to Volterra integral equations, Fixed Point Theory Appl., vol. 196, pp. 1-16, 2013.

16. N. Hussain, Lj. Ćirić, and N. Shafqat, Random fixed points for $\Psi$-contractions with application to random differential equations, Filoma, vol. 31 no. 3, pp. 759-779, 2017.

17. S. Itoh, Random fixed point theorems with an application to random differential equations in Banach spaces, J. Math. Anal. Appl., vol. 67, pp. 261-273, 1979.

18. A. Kaewcharoen, PPF depended common fixed point theorems for mappings in Banach spaces, J. Inequalities Appl., vol. 2013, pp. $1-14,2013$.

19. M. A. Kutbi, N. Hussain, and S. Khaleghizadeh, New PPF dependent fixed point theorems for Suzuki type GF-contractions, J. Function Spaces, vol. 2015, pp. 1-13, 2015.

20. J. J. Nieto, and R. Rodríguez-López, Contractive mapping theorems in partially ordered sets and applications to ordinary differential equations, Order, vol. 22, pp. 223-239, 2005.

21. V. Parvaneh, H. Hosseinzadeh, N. Hussain, and Lj. Ćirić, PPF dependent fixed point results for hybrid rational and Suzuki-Edelstein type contractions in Banach spaces, Filomat, vol. 30, no. 5, pp. 1339-1351, 2016.

22. R. A. Rashwan, and H. A. Hammad, Random fixed point theorems with an application to a random nonlinear integral equation, Journal of Linear and Topological Algebra, vol. 5, pp. 119-133, 2016.

23. R. A. Rashwan, and H. A. Hammad, Random common fixed point theorem for random weakly subsequentially continuous generalized contractions with application, Int. J. Pure Appl. Math., vol. 109, pp. 813-826, 2016.

24. P. Saipara, W. Kumam, and P. Chaipunya, Modified random errors S-iterative process for stochastic fixed point theorems in a generalized convex metric space, Stat., Optim. Inf. Comput., vol. 5, no. 1, pp 65-74, 2017.

25. W. Sintunavarat, and P. Kumam, PPF dependent fixed point theorems for rational type contraction mappings in Banach Spaces, J. Nonlinear Anal. Optim. Theory \& Appl., vol. 4, pp. 157-162, 2013.

26. A. Spacek, Zufăllige Gleichungen, Czechoslovak Math. J., vol. 5, pp. 462-466, 1955. 